\title{
CREATING VALUE WITH BUSINESS ANALYTICS EDUCATION
}

\author{
Ozay Ozaydin \\ Faculty of Engineering \\ Dogus University \\ Istanbul, Turkey \\ E-mail: oozaydin@dogus.edu.tr \\ Fusun Ulengin \\ School of Management \\ Sabanci University \\ Istanbul, Turkey \\ E-mail: fulengin@sabanciuniv.edu
}

\section{ABSTRACT}

Keywords: business analytics, education, ahp

\section{Introduction}

The third millennium is expected to be a challenge with its highly dynamic circumstances that is driven by the combination of population growth, resource depletion and rising expectations of given standards. This will force the firms to restructure and to become leaner in order to compete successfully in the marketplace, both domestically and globally (Manyika et al., 2011). Finding ways to provide competitive advantages will continue to be a great concern for many countries. In recent years, business analytics has become increasingly important in the world of business, particularly as organizations have access to more and more data (Evans, 2014). Business analytics has been recently adopted across a variety of organizations as an important business function that adds value. Researches conducted show that high-performing businesses use five-time more analytics in their strategic decisions when compared to low-performing businesses (Davenport and Harris, 2007). Davenport and Harris (2007) define analytics as "the extensive use of data, statistical and quantitative analysis, explanatory and predictive models, and fact-based management to drive decisions and actions". Based on this definition, analytics consists of descriptive, predictive and prescriptive modeling. The basic drivers of analytics movement are availability of data, improved statistical software, the adoption of a process orientation by many organizations and the presence of technically literate managers (Liberatore and Luo, 2011). Business analytics include the application of more sophisticated mathematical, statistical, and econometric methods to test and verify causal relationships within an organization 
Many organizations collect much data on their performance in various business areas. Business analytics support managers in understanding and structuring business Dynamics, its allows fort he testing of strategy strength, it can help to speed up task execution and can reduce the risk of time-consuming mistakes by identifying previous causes of errors. The application of business analytics can improve management's understanding of market behavior and customer behavior and it can allow for more formal reporting in the decision-making process (Klatt et al., 2011).

This increased use of analytics has led various Professional associations to evaluate the impact of analytics on their profession (Liberatore and Luo, 2011). Liberatore and Luo (2010) focus on the origin of analytics, its scope and drivers. In fact, according to Lauder (2011) corporations typically rate their management workers as A, B, and C players. A players are the technically literate managers who are crucial to the future of the company, the B players are the "engine house" who get things done but generally do not lead the charge and the $\mathrm{C}$ players are the routine knowledge workers. Leading transitional corporations gravitate towards the graduates of global elite universities because they are believed that they have the best students (Lauder, 2011). Business graduate programs have made a number of innovations in an attempt to enhance the quality of their offerings in a way to increase the number of students that will be potential A level of workers (Peterson et al., 2006).

Over the past two decades, the advancement of business analytics has brought powerful instruments to increase the competitive advantage of organizations. The analytical tools such as decision support systems, expert systems, and data mining systems provide valuable support to strategic planning and performance management of companies (Klatt et al., 2011). To avoid bounded or inefficient decisions, business analytics must provide decision makers with reliable information about key performance drivers.. Increased business competition requires even more rapid and sophisticated information and data analysis. The administration and faculty should be sensitive to the requests and needs of the next generation of business leaders. The practice of management has changed considerably in the past 50 years, yet MBA education, to a great extent, has not. Despite the explosive growth of business globally, MBA students are still primarily taught the same way as they were in the 1960's and 1970's. While many universities have made significant changes to their MBA curricula in recent years, these changes, largely experimental ones, have experienced both success and failure (Jain and Stopford, 2011). Mintzberg (2004) has referred particularly to the weaknesses in the MBA programs when it comes to fostering managers Others such as Crainer and Dearlove (1999, p.105) have argued that business schools simply provide a mechanism for selecting elites: "people don't go to the top business schools just for the learning; they go to join an elitist club, which has little to do with their ability as managers (Engwall (2007).

According to Kiron and Shockley (2011), the organizations can be categorized into three levels according to their analytics proweness. Aspirational companies 
are basic analytics users. They rely on analytics for financial and supply chain management and primarily use spreadsheets and structured, siloed data that support their targeted activities. Experienced companies rely on analytics to guide strategy as well as day-to-day activities in marketing and operations. This group has also experience with analytic tools such as data visualization and advanced modeling techniques. Transformed companies, on the other hand, are strong and sophisticated analytics users. They rely on analytics in most activities to guide both day-to-day operations and strategy. According to the survey that they conducted in 2011 the percentage of Experienced and Transformed organizations reporting competitive advantage from analytics grew substantially, whereas Aspirationals slipped by 5\%. This shows the gaining importance of analytics usage for increasing competitive advantage. In effect, the most advanced users of analytics typically have strong data-oriented culture that supports and guides analytics use. Transformed and Experienced users which are organizations that have moved beyond baseline analytics are disproportionately using analytics to focus on the future, on the customer and on increasing efficiencies at greater depth and scope than Aspirationals. The organizations that excel at using analytics to create a competitive advantage must also excel at information management and analytics expertise. The survey showed that the majority of Transformed organizations are strong on both data-oriented culture, information management and analytics expertise.

Business analytics can only be applied when the organizational culture supports the idea of generating results by using historical data. Additionally, organizations should grant their employees the opportunity to develop personally within business analytics. Education should be redesigned to generate adequate skills in the workforce.

Analytics and decision automation are among the most powerful tools for improving decision-making. A growing number of firms are embracing the former both strategically and tactically building competitive strategies around their analytical capabilities and making decisions on the basis of data and analytics (Davenport, 2006, 2009)

Senior executives are not the only people needed to build analytical business. Almost any employee can play a vital role as an analytical leader (Davenport and Harris, 2010).

Business analytics is the use of data, information technology, statistical analysis, quantitative methods and mathematical or computer-based models to help managers gain improved insight about their business operations and make better, fact-based decisions (Evans, 2014). Various research studies have discovered strong relationships between a company's performance in terms of profitability, revenue and shareholder return and its use of analytics. Top performing organizations are three times more likely to be sophisticated in their use of analytics than lower performers (Davenport and Harris, 2007). Thus understanding the capabilities and techniques of analytics and investigating the different business analytics graduate program offered worldwide and than

International Symposium of
the Analytic Hierarchy
Process


revealing the analytics needs of the managers of industries should be the basic stages to develop a suitable curriculum that will be vital to managing in today's business environment.

The modern evolution of analytics began with the introduction of computers in the late 1940s and their development through the 1960s and beyond (Evans, 2014). In 1958, an IBM researcher Hans Peter Luhn coined a term Business intelligence to define the facilitation of the collection, management, analysis and reporting of data. Much of modern business analytics stems from the analysis and solution of complex decision problems using mathematical or computer based models based on a discipline that is known as operations research or management science, with more emphasis on business application in the latter. Decision support systems, INFORMS, The Institute for Operations Research and Management Sciences is the leading Professional society devoted to analytics. In 1960, the combination of business intelligence concepts with management science models resulted with analytical-based computer systems called decision support systems. This system includes Data management, model management and communication system. Data management consists of databases for storing data and allows the user to input, retrieve, update and manipulate data. Model management consists of various statistical tools and management science models to allow the user to build, manipulate, analyze and solve models. Finally, the communication system provides the interface for the user to interact with the data and model management components.

\section{Literature Review}

Although there are many researches and surveys conducted to analyze the current state of business analytics in United States, unfortunately there is a lack of such studies in other parts of the world. In this research, we aimed to analyze the perception and use of business analytics in Turkish firms. The second section highlights the research methodology used in this study in order to analyze the current status of business analytics perceptions in Turkey's business environment. Section 3 provides the results of cluster analysis that classify the existing Business Analytics graduate programs in the world according to their similarity. Section 4 evaluates the results of the survey conducted with Turkish firms being ranked as top Fortune 500 companies of Turkey. Finally the relative weights assigned by the survey respondents to different clusters of Business Analysis graduate program curriculum are calculated using Group AHP and the individual as well as group consistencies are calculated.. Finally conclusions and further suggestions are provided.

\section{Hypotheses/Objectives}

This section will be concluded in the final version

\section{Research Design/Methodology}


In order to develop a suitable business analytics graduate program suitable to the needs of the organizations a 3-step methodology is conducted. In the first stage; a worldwide analysis of the curriculum of *** business analytics programs is made and based on the description of the offered courses, the courses are grouped into 5 groups.

In the second stage; a cluster analysis is conducted to group the programs according to their similarity in terms of the offered courses. In the third stage an online survey is conducted top 500 Turkish companies according to Fortune 500 classification.

A group AHP analysis is conducted in order to reveal their relative preferences concerning those clustered programs. Additionally additional courses not offered in any of the clusters but thought to be important for Turkish business managers are also asked from the CEOs. The consistency as well as sensitivity are also realized. Figure 1 shows the basic steps of the methodology.

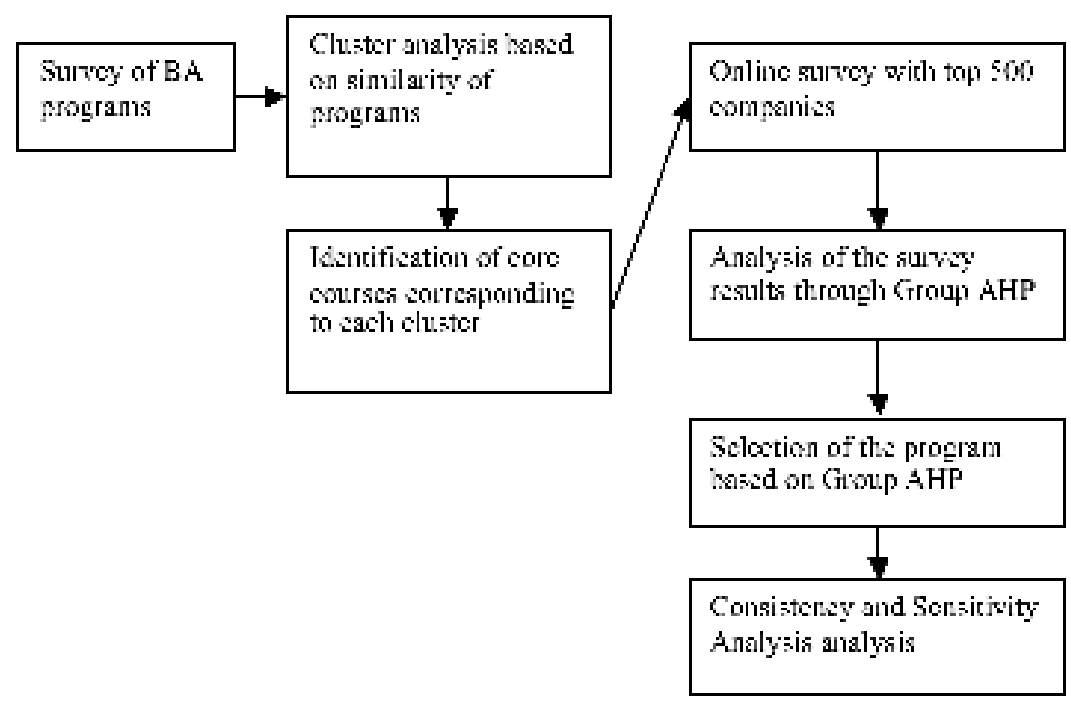

Figure 1. Flowchart of the Proposed Methodolgy

\section{Data/Model Analysis}

Cluster analysis, which can also be called as segmentation analysis or taxonomy analysis, involves grouping similar objects into mutually exclusive subsets referred as clusters (Hair et al., 2009). Cluster Analysis is a multivariate data analysis technique used in grouping objects according to the characteristics they possess. Clusters are formed in such a way that objects in the same cluster are very similar and objects in different clusters are very different. The cluster definition problem is NP-complete. As a result, an optimum does not exist. A number of heuristic methods including agglomerative techniques, which are the mostly widely known and used, have been built for this purpose. All hierarchical agglomerative heuristics begin with $\mathrm{n}$ clusters where $\mathrm{n}$ is the number of 
observations. Then, the two most similar clusters are combined to form n-1 clusters. On the next iteration, n-2 clusters are formed with the same logic and this process continues until one cluster remains. Only the rules used to merge clusters differ in the various hierarchical agglomerative heuristics. The "Simple Linkage" approach merges the clusters by finding the minimum distance between one observation in one cluster and another observation in the second cluster. "Furthest Neighborhood", in contrast, takes the furthest distance between two observations, while "Average Linkage" takes the average distance of the observations belonging to each cluster and merges them with a minimum average distance between all pairs of observations in the respective clusters. In Ward's method, on the other hand, the distance is the ANOVA sum of squares between the two clusters summed over all variables (Hair, 2009). The "centroid" and "median" methods can produce a cluster tree that is not monotonic. This occurs when the distance from the union of two clusters, $r$ and $s$, to a third cluster is less than the distance from either $r$ or $s$ to that third cluster. In this case, sections of the dendogram change direction (Hair, 2009).

Although all hierarchical methods successfully define clusters for compact and isolated data, they generally fail to accurately provide defined clusters for "messy" data. The major issue with all clustering techniques is how to select the number of clusters. Different clustering methods may lead to different clusters and the differences are generally due to the inherent characteristics of the methodology used. In fact, there is no single methodology that can be recommended in selecting the most appropriate number of clusters and the most suitable clustering method. That is why cluster analysis is generally accepted to be more of an art than a science (Milligan, 1980).

\section{Limitations}

This section will be concluded in the final version

\section{Conclusions}

This section will be concluded in the final version

\section{Key References}

Davenport T. Harris J.G. (2007) Competing on Analytics: The New Science of Winning, Harvard Business School Press, Boston, MA, ISBN:

Davenport, T., Harris, J.(2010) Analytics at Work, Smarter Decisions, Better Results. Harvard Business School Press Jain S.C., Stopford J. (2011) Revamping MBA programs for global competitiveness, Business Horizons, 54, pp.345-353.

Hair J.F.Jr., Black W.C., BabinB.J., AndersonR.E. (2009) Multivariate Data Analysis (7th Edition), Prentice Hall, ISBN: 978-0138132637. 